\title{
Video Article \\ Long-term Continuous EEG Monitoring in Small Rodent Models of Human Disease Using the Epoch Wireless Transmitter System
}

\author{
Andrew Zayachkivsky ${ }^{1}$, Mark J. Lehmkuhle ${ }^{2}$, F. Edward Dudek ${ }^{2}$ \\ ${ }^{1}$ Department of Neurosurgery, Yale University School of Medicine \\ ${ }^{2}$ Department of Neurosurgery, University of Utah
}

Correspondence to: Andrew Zayachkivsky at avzay55@gmail.com

URL: https://www.jove.com/video/52554

DOI: doi: $10.3791 / 52554$

Keywords: Neuroscience, Issue 101, Epilepsy, Seizures, Wireless, Pre-Clinical, Rat, Mouse, Hypoxia, Ischemia, Neonate

Date Published: 7/21/2015

Citation: Zayachkivsky, A., Lehmkuhle, M.J., Dudek, F.E. Long-term Continuous EEG Monitoring in Small Rodent Models of Human Disease Using the Epoch Wireless Transmitter System. J. Vis. Exp. (101), e52554, doi:10.3791/52554 (2015).

\section{Abstract}

Many progressive neurologic diseases in humans, such as epilepsy, require pre-clinical animal models that slowly develop the disease in order to test interventions at various stages of the disease process. These animal models are particularly difficult to implement in immature rodents, a classic model organism for laboratory study of these disorders. Recording continuous EEG in young animal models of seizures and other neurological disorders presents a technical challenge due to the small physical size of young rodents and their dependence on the dam prior to weaning. Therefore, there is not only a clear need for improving pre-clinical research that will better identify those therapies suitable for translation to the clinic but also a need for new devices capable of recording continuous EEG in immature rodents. Here, we describe the technology behind and demonstrate the use of a novel miniature telemetry system, specifically engineered for use in immature rats or mice, which is also effective for use in adult animals.

\section{Video Link}

The video component of this article can be found at https://www.jove.com/video/52554/

\section{Introduction}

The oldest - and still the most widely used - technique for recording biopotentials in the brain is the electroencephalogram (EEG). It is used clinically for neurologic abnormalities, including seizure detection ${ }^{1}$, localization of seizure foci ${ }^{2}$, and diagnosis of concussion ${ }^{3,4}$. This technique is also widely used to provide fundamental information about the mechanisms of sleep and to diagnose sleep disorders ${ }^{5,6}$.

As in the clinical diagnosis of the epilepsies, the EEG has become indispensable for translational research in animal models of both genetic and acquired epilepsy. In current research applications, "wired" or "tethered" recordings are standard, and are routinely performed in adult rodents for weeks at a time ${ }^{7}$. However, electrical noise, movement artifacts, and the risk that tethered animals will injure themselves by pulling on the cable have long compromised these experiments. Thus, to improve experimental conditions and success rates, we need to develop new technologies that would allow for elimination of the wired interface between the animal and instrumentation. The most obvious area of development is design and implementation of telemetry systems that allows for high-quality recordings, while maintaining a long useful life and minimizing discomfort for animal subjects. Reducing the physical size of these devices will enable translational research in neonatal and juvenile rodent models of neurological disorders.

Low channel-count EEG recordings in rats are employed extensively to develop new therapies to suppress epileptic seizures capable of translation to humans. Recordings from one or more sites for a prolonged period open many possibilities for using rodent models of epilepsy in translational research. Much of the contemporary research in this field aims to block the occurrence of chronic seizures or the development of epilepsy (i.e., epileptogenesis), and such research efforts require extensive if not continuous EEG monitoring to assay the effectiveness of the proposed therapy ${ }^{8}$; a small, simple, telemetric system with one, two, or four channels operating between $0.1-100 \mathrm{~Hz}$ per channel will strongly promote this type of translational research. Electrographic seizures often occur with minimal behaviors (certainly without convulsions), which limits the usefulness of assays based on behavioral seizures. The strategy of combining EEG recording and simultaneous video monitoring allows one the possibility of capturing every seizure; and furthermore, these analytical approaches may allow quantitative assessment of the interictal spikes that occur in the epileptic brain between "ictal" (or seizure) events ${ }^{9}$. Furthermore, the ability to obtain continuous high-quality lowartifact EEG recordings, for which the wireless technology is generally superior, will allow for development of use of computer-based algorithms for studying specific EEG waveforms (e.g., theta, gamma), as well as automatic detection of seizures, significantly reducing the workload of the experimenter.

The primary pre-clinical model for studying chronic epilepsy after brain injury is the adult rat or mouse, either through a chemo-convulsant (i.e., kainic acid or pilocarpine) or electrically induced status epilepticus (SE), which is followed by chronic epilepsy. Under these conditions, the severe convulsions associated with SE or the subsequent seizures in the epileptic animals can lead to injury from the animal tearing or pulling on the tether and loosening the screws that maintain the attachment of the headcap. Ultimately, it is this problem that usually terminates these 
experiments, and yet the need to obtain long-term high-resolution EEG records for experiments aimed at developing new therapies for chronic epilepsy is paramount. Additionally, housing, monitoring, and analyzing the data from long-term implanted animals is a considerable investment in both direct costs and investigator time; therefore, premature termination of the experiment can result in significant costs to the researchers. As these models of epilepsy progress, the seizures usually become more frequent and more severe ${ }^{10-12}$, increasing the likelihood that animals are injured, just as their usefulness for developing new therapies becomes greatest. These animals can routinely develop dozens of convulsive seizures per day, often occurring in clusters ${ }^{13}$.

Probably one of the most important developments in biomedical science has been the use of gene targeting in mouse models. This approach has allowed, and will continue to allow, the development of animal models of genetic epilepsy that reproduce actual human syndromes ${ }^{14-16}$. Genetic manipulations can be undertaken as proof-of-principle therapies to suppress epileptic seizures or even block the development of epilepsy after brain injury ${ }^{17-20}$. This type of research would benefit dramatically from the ability to perform high-throughput continuous recording of the EEG. Presently, it is possible to record from mice with either tethered or telemetry systems; however, the challenges of obtaining highquality, artifact-free recordings are substantially more difficult than rats, and often this requires various forms of backpacks that mice continuously attempt to remove. Stress could increase seizure severity, frequency and/or duration, and thus would ultimately modify the epilepsy of the experimental animals, thus confounding the study. A small, light-weight, low-profile miniature telemetry system will facilitate the recording of longterm EEG from genetic mouse models of human disease.

In addition to the above described problems, recording EEG in immature rodent models of disease has its own unique set of challenges. Immature animals can weigh as little as $6 \mathrm{~g}$ (P8 mouse) to $17 \mathrm{~g}$ (P6 rat). It is virtually impossible to make serial multi-day tethered EEG recordings due to increased stress from tether and inability to allow natural rearing of the pup by the dam. Until animals are weaned, they must remain in the care of the dam. The dam is prone to destroy any externalized connector assembly on the pup, terminate the pup, and in some cases terminate the entire litter. Furthermore, the immature rodent skull makes it difficult to mount any electrode pedestal to the skull with mechanical integrity. These challenges, unique to immature rodents, require a novel solution for making robust, long-term electrographic recordings. Here we focus on demonstrating the implantation and recording of EEG using a novel miniature wireless transmitter and present three proof-of-principle experiments as examples for use of the miniature wireless telemetry system: 1) the immature rat pup model of hypoxiaischemia, 2) adult mice treated with DFP to induce status epilepticus and subsequent spontaneous seizures, and 3) genetic model of vascular cavernous malformations which result in seizures and death in adult mice.

The miniature wireless telemetry system was designed to meet four main requirements: (1) minimally invasive surgical implantation; (2) compatibility for housing of rodent pups with the dam and littermates; (3) low power consumption of the unit, thus allowing for months of continuous monitoring without surgical re-implantation; and (4) ability to record high-quality EEG waveforms with minimal movement artifacts. The wireless transmitter weighs $<0.6,2.3$, and $4 \mathrm{~g}$ and is $<0.3,0.8$, and $1.4 \mathrm{~cm}^{3}$ depending on the battery with a footprint of $5 \times 7,7 \times 9$, or $7 \times$ $12 \mathrm{~mm}$ that easily mounts to the skull of the animal with cyanoacrylate gel. No bone screw anchors are needed to securely affix the device to the skull, reducing the number of holes that need to be drilled in the skull and the surgery time. The device is capable of amplifying two channels of EEG or local field potentials from deep brain structures, such as the hippocampus, for over 2 weeks, 2 months, or 6 months in this configuration. The small size of the wireless transmitter reduces risk of infection, increases animal mobility, and ultimately reduces morbidity and mortality that otherwise increases the time, money, and number of animals needed for an experiment. All of the electronics and battery are potted in medicalgrade epoxy that makes the device waterproof and tough, preventing the dam from chewing on the transmitter that could otherwise render the device inoperable. Unlike radio-frequency transmitters, the telemetry system uses capacitive coupling between the transmitter and a receiver antenna that sits below the animal cage, allowing the user to keep animals in standard rodent housing. Multiple channels of recording allow for the recording of multi-modal biopotentials, such as electrocardiogram and electroencephalogram. Animal models of co-morbidities will benefit by the ability to record biopotentials during behavior ${ }^{21-23}$. Combining behavior with EEG monitoring will provide researchers with a better tool for research and pre-clinical studies.

\section{Protocol}

Follow the institutional guidelines for animal care for surgical tool sterilization, and modify the protocol as necessary to comply with the guidelines and obtain approval by your institution's Institutional Animal Care and Use Committee (IACUC).

\section{Surgical Preparation}

1. Clean and prepare the transmitter to ensure safe and sterile surgery. Remove the transmitter from its anti-static packaging and either spray or soak in $70 \%$ ethanol. Rinse transmitter with sterile saline and place between sterile cotton sponges soaked in sterile saline or keep submerged in sterile saline.

2. Collect and sterilize the tools required for surgery; steam autoclave for sterilization. See table of materials and reagents for the list of surgical tools.

\section{Surgical Implantation}

1. Anesthetize animal and maintain anesthesia according to IACUC-approved protocol. At initiation and during surgery check the toe-pinch reflex every $15 \mathrm{~min}$. The lack of response indicates sufficient level of anesthesia.

1. For pups, use anesthesia by isoflurane $(4 \%)$ with $\mathrm{O}_{2}(100 \%)$. For adults, use ketamine $(100 \mathrm{mg} / \mathrm{kg})$ with $\mathrm{xylazine}(10 \mathrm{mg} / \mathrm{kg})$.

2. Fix position in stereotaxic frame. Place the ear bar tips in the auditory meatus. Do not excessively tighten ear bars as the skull is very soft in young rat pups. Secure the anesthesia nose cone.

1. Keep the animal warm during surgery by placing it on the heating pad set to $37^{\circ} \mathrm{C}$. In adult animals, apply lubricating ointment to the eyes of the animal. 
3. Sterilize incision site and maintain sterile surgical field.

1. Swab the scalp with alternating applications of $70 \%$ ethanol and betadine. Start in the center of the scalp and make increasingly wider concentric circles.

2. Cover the animal with drape and conduct the surgery over draped animal. Maintain the sterile surgical field by lining the surgical set-up with sterile drapes, spray equipment with $70 \%$ ethanol.

3. Wear sterile surgical gloves and gown (or as required by the institution). To help maintain sterile field, use a surgical assistant.

4. Make an incision on the scalp of the animal slightly behind the eyes along the midline, approximately $2 \mathrm{~cm}$. Use caution when inserting the scalpel as the skull is still very soft in young rat pups. Make a single cut so the incision bleeds less, and heals faster.

5. Expose the skull. Prepare a clean and dry area to maximize the bond between the transmitter and the bones of the skull. Use aneurism clips to grasp scalp.

1. Gently pull scalp away from midline at four corners. Look for anatomical landmarks such as bregma and lambda in the skull. Remember skull bones are not fused in animals at this age. Use the Paxinos atlas of stereotaxic coordinates to find the correct location for the burr hole.

6. Use a Dremel-type tool with a burr-type drill bit. Create two burr holes in desired recording positions with the holes being bigger than $300 \mu \mathrm{m}$ in diameter. Place the burr hole for the reference electrode over the cerebellum behind the lambda of the skull.

7. Ensure that the wires on the transmitter are aligned with the burr holes. If the electrode wires are not aligned, glue contamination of the electrodes is likely, and will result in poor signal. To align the wires, check the fit of the transmitter and gently bend electrodes to line up over the intended sites for burr holes.

8. Trim electrode leads. Use surgical scissors to trim the electrodes to the desired length. The electrode depth is important for the type of recording required for the experiment (i.e., place the electrodes above dura for EEG recordings, or use stereotaxic coordinates for defined brain structures).

9. Liberally apply cyanoacrylate on the base of the transmitter to cover the area making sure to avoid coating the electrodes. Cyanoacrylate glue is an electrical insulator, contaminating electrodes with glue will result in no signal.

1. If recording from deep brain structures, mount the transmitter on the cannula holder and place it in the stereotaxic arm for $z$-axis control. Lower the transmitter using the stereotaxic arm to appropriate depth and place cyanoacrylate gel around the transmitter.

10. Thoroughly dry skull before placing transmitter to ensure strong adhesive bond. Apply transmitter coated with cyanoacrylate to the skull. Take care to align electrodes with corresponding burr holes.

1. Try to avoid damaging major vascular structures. Hold the transmitter in place with slight pressure for one minute. Use slight pressure to form a strong bond between the transmitter and the skull.

11. Apply additional cyanoacrylate, enough to completely seal transmitter/skull interface. To ensure a good fit and strong bond, maximize the surface area of the glue that contacts the skull. Apply the cyanoacrylate adhesive in a circle around the transmitter, making sure both skull and the wall of the transmitter are covered.

12. Apply chemical accelerant $(0.1 \mathrm{ml})$ through a syringe around the cyanoacrylate at the base of the implanted transmitter. Use accelerant sparingly, taking care not to apply to adjacent tissue.

Note: Chemical acceleration of the cyanoacrylate curing ensures that the strong bond between the transmitter and the skull is formed quickly. Cyanoacrylate accelerant is useful to speed curing of adhesive, but is not necessary.

13. Remove the accelerant by washing the area thoroughly with sterile saline. Cyanoacrylate accelerator may cause tissue irritation if not washed from the area of the incision. To wash the area, fill a $1.0 \mathrm{ml}$ syringe with sterile saline and irrigate the area through a syringe needle. Generally $0.5 \mathrm{ml}$ of saline is enough to wash out the accelerator.

14. Suture the skin around the base of the transmitter, but do not cover the transmitter. Top of transmitter must be above skin to efficiently transmit neural signals. Skin should be reasonably tight around the transmitter and the glue around the unit. Use Vicryl or silk suture (soft thread); skin in immature animals is soft and is easily damaged if soft sutures are not used. For adult animals, use any suturing material.

15. Remove animal from stereotaxic frame and place on heated blanket for recovery.

16. Ensure animals are warm $\left(37^{\circ} \mathrm{C}\right)$ and ambulatory (i.e., completely recovered) before returning to the dam. Ensure that the animal is hydrated by pinching the skin on the animal's back (if the animal is dehydrated, the skin will remain deformed). If animal is dehydrated, administer subcutaneous injection of lactated Ringer's buffer. Do not leave the animal unattended until it has regained sufficient consciousness to maintain sternal recumbency.

1. Administer buprenorphine $(0.05 \mathrm{mg} / \mathrm{kg})$ to animals for post-surgical pain management and a sub-cutaneous injection of $0.1 \mathrm{ml}$ bupivacaine around the injection site.

Note: From start to finish the entire procedure should be completed in 5-10 min for animals of this age (postnatal day 6). Surgical time may take longer for older animals.

\section{Care and Housing}

Note: Some dams may not tolerate pups implanted with the device. Dams may need to be selected who are tolerant. It is acceptable for the dam to move pups around the cage by picking them up by the transmitter.

1. Once animals are weaned, singly-house them to avoid the removal of the devices from their cage mate.

2. Euthanize animals by lethal dose of pentobarbital $(25 \mathrm{mg} / \mathrm{kg}$ ) or isoflurane (in a bell jar) when signs of distress are present

3. Note, some animal housing cages with wire inserts may interfere with the implanted transmitters. Be sure to check the height of the wire insert to make sure that animals cannot get the transmitter caught between the 'bars' of the wire insert. Consult your veterinarian for help. 


\section{Recording EEG}

1. Place the animal in a cage by itself or co-housed with littermates and the dam. However, place only one implanted animal in a single cage. Do not leave pups alone in the recording chamber for more than $2 \mathrm{hr}$. Monitor the animals for signs of distress and dehydration.

2. Connect the provided power supply to the receiver base and verify the power light is illuminated. Connect the receiver base to a data acquisition system using (Bayonet Neil-Concelman) BNC cables.

3. Place the animal cage on top of the receiver base (Figure 2). The "signal" light should illuminate indicating a transmitter has been detected. Data may now be recorded.

4. To record data, connect the receiver base to an analogue-to-digital converter and connect the converter to a computer (Figure 1).

5. Set the sampling rate of the recording. Ensure the data is sampled properly. Select at least $250 \mathrm{~Hz}$ sampling rate (500 $\mathrm{Hz}$ recommended) for recording (bandwidth of the transmitter is $0.1-100 \mathrm{~Hz}$ ).

6. Save digitized data and analyze using signal-processing software packages such as Matlab.

\section{EEG Analysis - General}

1. Perform FFTs (fast Fourier transforms) to transform temporal EEG data to the frequency domain from $0-100 \mathrm{~Hz}$.

2. Perform an estimate of power spectral densities (PSDs) from the FFT using 256 Hann-window segments based on the Welch method and normalized by $10 \times \log _{10}(P S D)$. Power spectra show the specific frequencies that dominate the EEG signal over the desired time period.

3. Group data across animals by taking the mean of the PSD from each animal over time-matched treatments. Create $95 \%$ confidence intervals by $1.96 \times$ mean(PSD)/square root $(n)$ where $n$ is the number of animals (PSD traces). Plot the mean and $95 \%$ confidence intervals of the data to generate a quantitative report of the entire frequency content of the EEG across cohorts of animals such as comparing treated groups vs. control groups.

\section{Perinatal Hypoxia-ischemia (HI) Model Protocol}

1. Anesthetize the P6 - 7 rat pup using isoflurane anesthesia ( $4 \%$ with $100 \% \mathrm{O}_{2}$ ) by placing the animal in an anesthesia box (box with input from anesthesia vaporizer). At initiation and during surgery check the toe-pinch reflex every $15 \mathrm{~min}$. The lack of response indicates sufficient level of anesthesia.

2. Place the pup on its back, expose the neck and scrub with alternating applications of $70 \%$ ethanol and $10 \%$ betadine. Repeat the ethanol/ betadine scrub 3 times.

3. Make a $1 \mathrm{~cm}$ incision in the skin of the neck with scissors at the midline of the neck. Lift the skin with forceps and make the cut with scissors. Take care not to cut the muscle tissue when making the incision.

4. Use blunt dissection technique to expose carotid artery. To perform blunt dissection, use two pairs of blunt-nose forceps. Insert the tips into the tissue and let the spring action of the surgical instrument spread the tissue. Repeat until carotid artery is exposed. Identify the carotid artery by a bright red color and the presence of visible pulse.

5. Separate carotid artery from the vagus nerve using blunt forceps. Insert the blunt-tipped forceps between the artery and the nerve. Release the forceps and let spring action of the tool separate the carotid from the vagus nerve.

6. Place aneurism clamps $4-5 \mathrm{~mm}$ apart on the carotid artery. Take care to not damage the artery with the clamps by avoiding rapid movements

7. Cauterize the carotid artery between the aneurism clamps. To cauterize the artery, touch the artery between the clamps with a hot cauterizer tip. After the artery is cut, ensure both ends are properly cauterized to avoid hemorrhage.

8. Remove the clamps, close the neck incision with 3 sutures. Only suture the skin, take care not to suture the muscle tissue.

9. Allow the animal to recover for $1 \mathrm{hr}$. Monitor the animal's breathing and bleeding from the neck. If bleeding is present, do not subject the animal to $\mathrm{HI}$ (step 6.10).

10. Place the animal in a temperature controlled chamber at $37^{\circ} \mathrm{C}$ and continuously introduce $8 \% \mathrm{O}_{2} / 92 \% \mathrm{~N}_{2}$ mixture into the chamber for 2 hr.

\section{Representative Results}

We developed and implemented the concept of recording EEG from a single adult rodent, schematized in Figure 1. For the IACUC approval process, the design must integrate well into existing institutional animal facilities; therefore, the system was designed to be easily installed in a standard animal facility without use of additional space: the animal is housed in a regular "animal facility-issue" housing cage that is placed inside of a receiver with an integrated Faraday cage to reduce electrical noise. The signal from each receiver base is conducted by wires to a digitizer that is connected to a computer (Figure 1). A single computer is needed to collect data from up to 32 animals recorded concurrently, depending on the capability of the user's data acquisition system. This type of setup consumes little power and produces little heat, a feature compatible with climate-controlled animal facilities. Data can be displayed in real-time on the monitor, allowing experimental monitoring, and stored longterm on external hard-drives (10 TB storage unit).

In order to minimize damage by litter-mates and pup cannibalization by the dam, we tested various transmitter form factors. The final design was a domed cylinder; a shape difficult for rats to bite and damage. An individual transmitter on the skull of an adult rat is shown in Figure 2A and an early version of high-density (32 animal) receiver bases and recording rigs in which standard rodent housing is placed is shown in Figure 2B. Power efficiency was an extremely important consideration; we chose capacitive coupling as a data transmission protocol. The following design allows for recording continuous EEG for over 6 months depending on battery capacity (Figure 2A). Mice as young as postnatal day 12 (P12, Figure 3A) and rats as young as P6 (Figure 3B) tolerate the transmitter quite well. Adhering the transmitter to the skull with cyanoacrylate enables animals to grow with the transmitter into adulthood (Figure $3 \mathrm{C}$ ), while maintaining continuous acquisition of EEG data.

The unique miniaturized form factor of the transmitter and wireless interface lends itself for work with animal models of neo- and perinatal conditions. The data in Figure 4 shows two-channels of EEG recording of sub-acute seizure activity that follows hypoxic-ischemic $(\mathrm{HI})$ infarct (carotid artery ligation followed by $2 \mathrm{hrs}$ of hypoxia with $8 \% \mathrm{O}_{2}$ mixture) in a P7 Sprague-Dawley rat pup ${ }^{13}$. The HI treatment causes a large 
lesion in the hemisphere ipsilateral to the ligated carotid. Here, the recordings show a cluster of two seizures generalized over both hemispheres of the injured brain. The black trace depicts EEG activity in the hemisphere contralateral to the lesion, the blue trace shows EEG in the ipsilateral hemispheres (i.e. in the area of the lesion). While the seizure activity is present in both hemispheres of the brain, the ispilateral hemisphere shows EEG background suppression, which is indicative of ongoing brain damage ${ }^{21}$.

Status epilepticus can be induced in adult rats by injecting the animals with the organophosphate, DFP ${ }^{22,23}$. The data in Figure 5 show repetitive EEG discharges, which are indicative of status epilepticus (see temporal expansions Figure 5A, B). Below the sample traces, the time course of status epilepticus over 12 hrs has been analyzed with a non-linear mixed effects model that quantifies the intensity of the seizures over time. The severity of status epilepticus is defined by EEG power in the gamma band $(20-60 \mathrm{~Hz})$. Here, the above described power was averaged across 12 animals and plotted over $12 \mathrm{hr}$ with 95\% confidence intervals. The data shows a marked increase in gamma power within the first hour of DFP treatment, which persists over $12 \mathrm{hr}$ during which animals were continuously monitored. The following method of analysis allows for a quantitative measure of the severity of acute status epilepticus, a phenomenon previously analyzed primarily with behavioral measures. We include this analysis technique as an example, because it utilizes power computation in classic EEG bands and has been extensively used in preclinical studies for testing the efficacy of anticonvulsant medications in our laboratory ${ }^{24-26}$. Possibly the most valuable aspect of making continuous, uninterrupted wireless recordings with the wireless telemetry is the ability to record abnormal spontaneous events that occur with low incidence. These types of data demonstrate the broad utility of the wireless transmitter system.

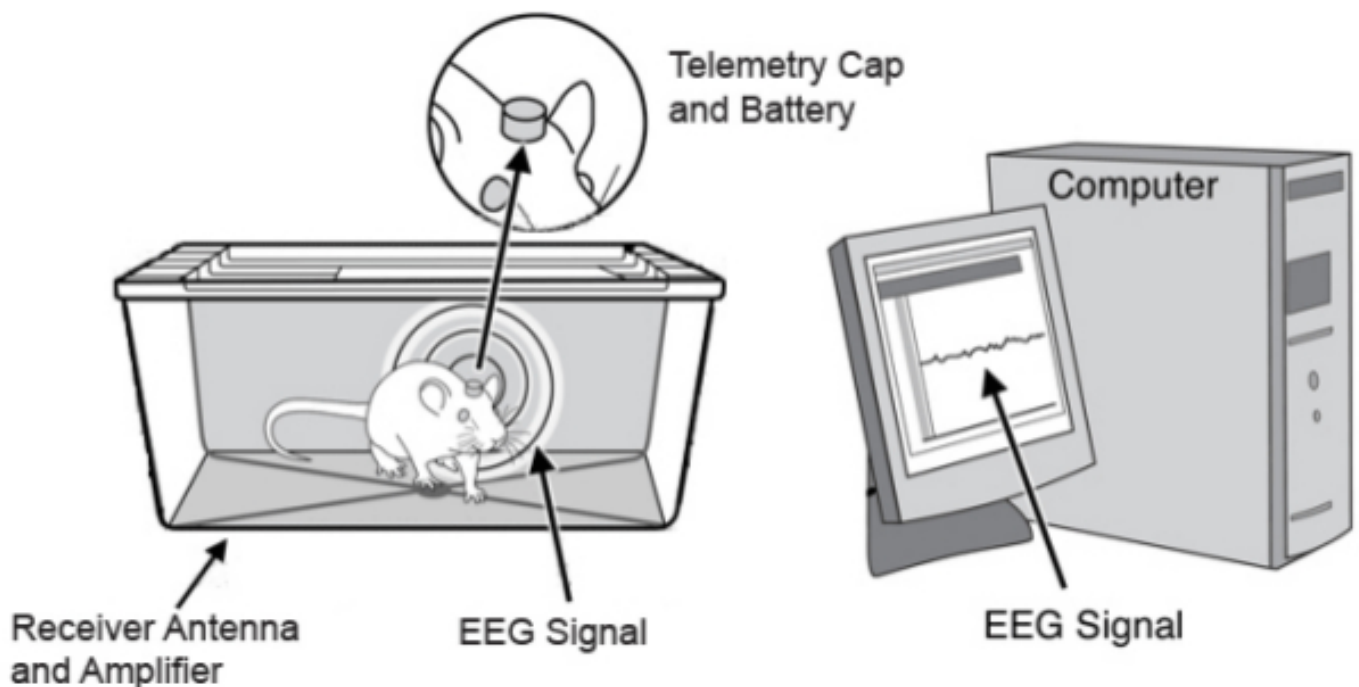

Figure 1: Schematic of the Epoch recording system. The wireless recording system consists of two components: 1) a wireless skull-mounted transmitter that amplifies the biosignal, and 2) a receiver plate placed beneath standard rodent housing. The output of the receiver base is an analog signal consisting of the demodulated biosignal amplified to a maximum of $4 \mathrm{~V}$ peak-to-peak. This signal can then be fed into a data acquisition system for recording. 

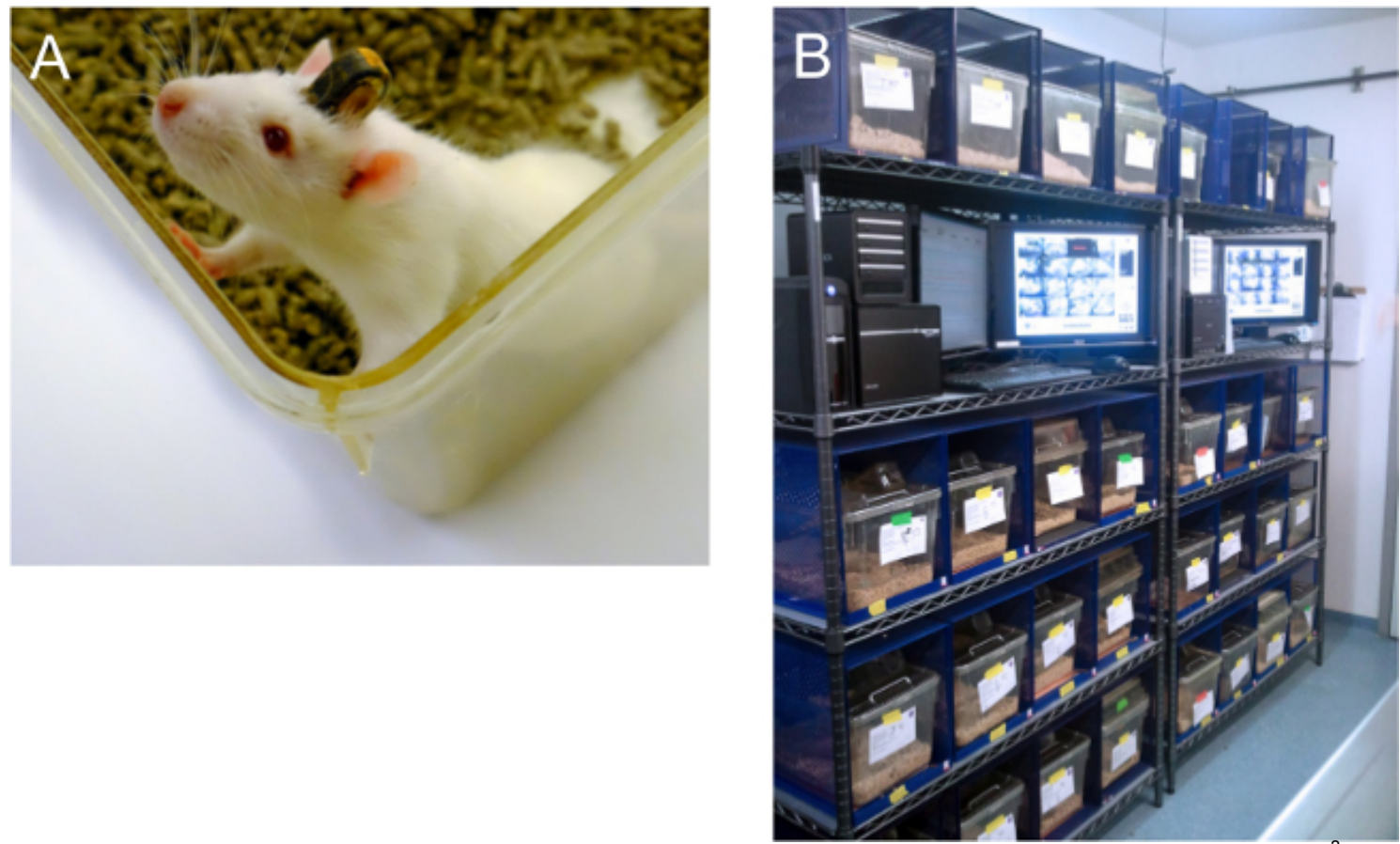

Figure 2: The transmitter and receiver. This particular wireless transmitter (A) weighs $4 \mathrm{~g}$ and displaces $<1.4 \mathrm{~cm}^{3}$ of volume and with a footprint of $7 \times 12 \mathrm{~mm}$ is easily mounted to the skull of rats and mice. The transmitter can amplify 2 channels of biopotentials for up to 6 months after which the battery is drained. Larger batteries can be used for longer recording time. Animals are placed in standard rodent caging on top of the Epoch receiver (B). Shown at right is an early example of two separate recording rigs each capable of recording from 16 animals simultaneously demonstrating the relatively small footprint (2' x 4', ca. $60 \mathrm{~cm} \times 120 \mathrm{~cm}$ ) of each of the recording rigs. 

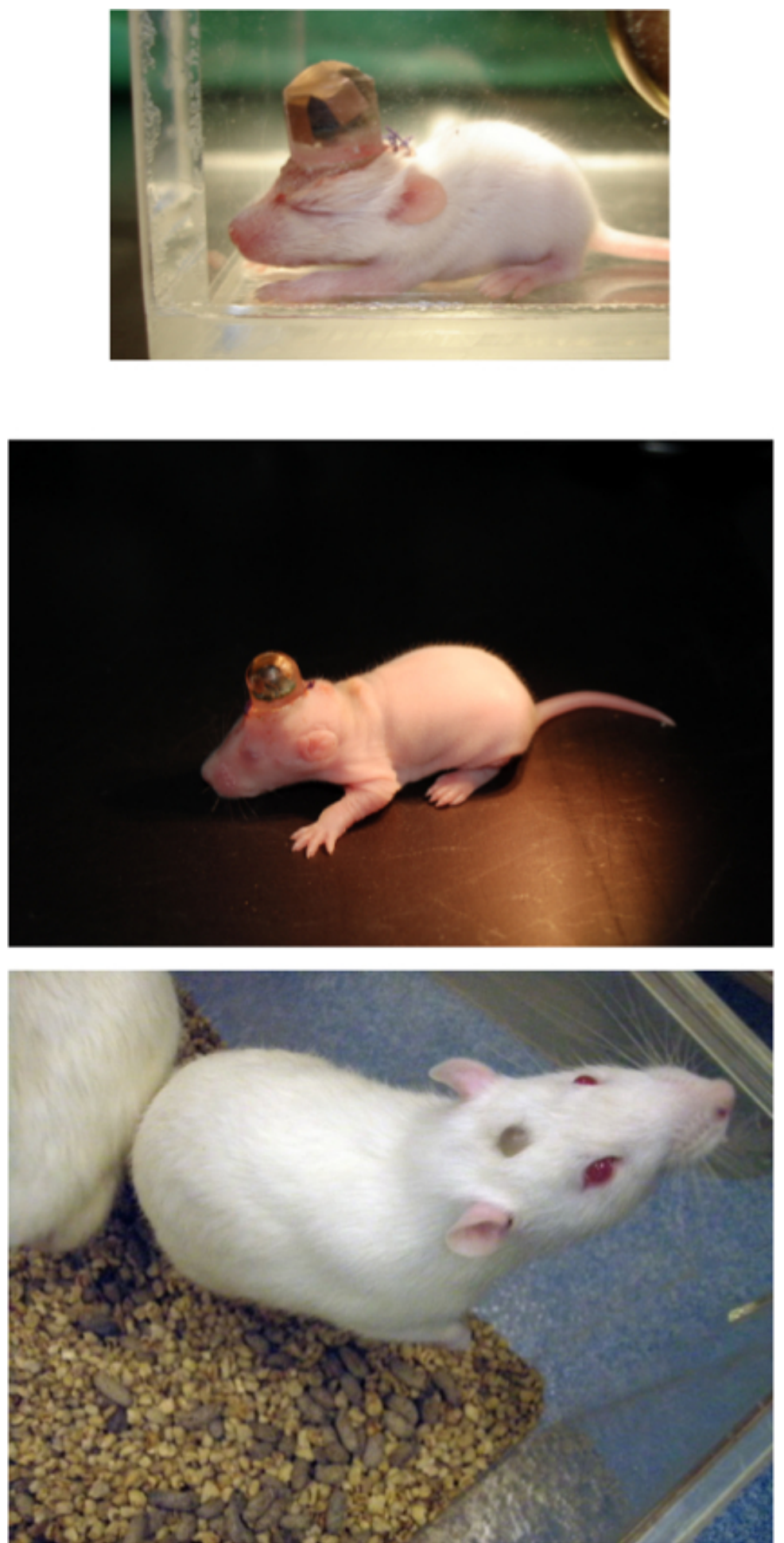

Figure 3: Implanting the wireless transmitter in rats and mice. The transmitter enables continuous EEG recordings for up to 6 months in mice as young as postnatal day 12 (P12, top). The middle photograph is of a P7 rat pup implanted with the miniature transmitter. The transmitter stays firmly attached to the skull as the animal matures. The animal at bottom is P280 and was implanted with a sham transmitter at age P7. The system enables simultaneous and continuous EEG recordings from multiple animals aged P7 through weaning, reducing the number of litters needed for pre-clinical, long-term, EEG monitoring studies. 


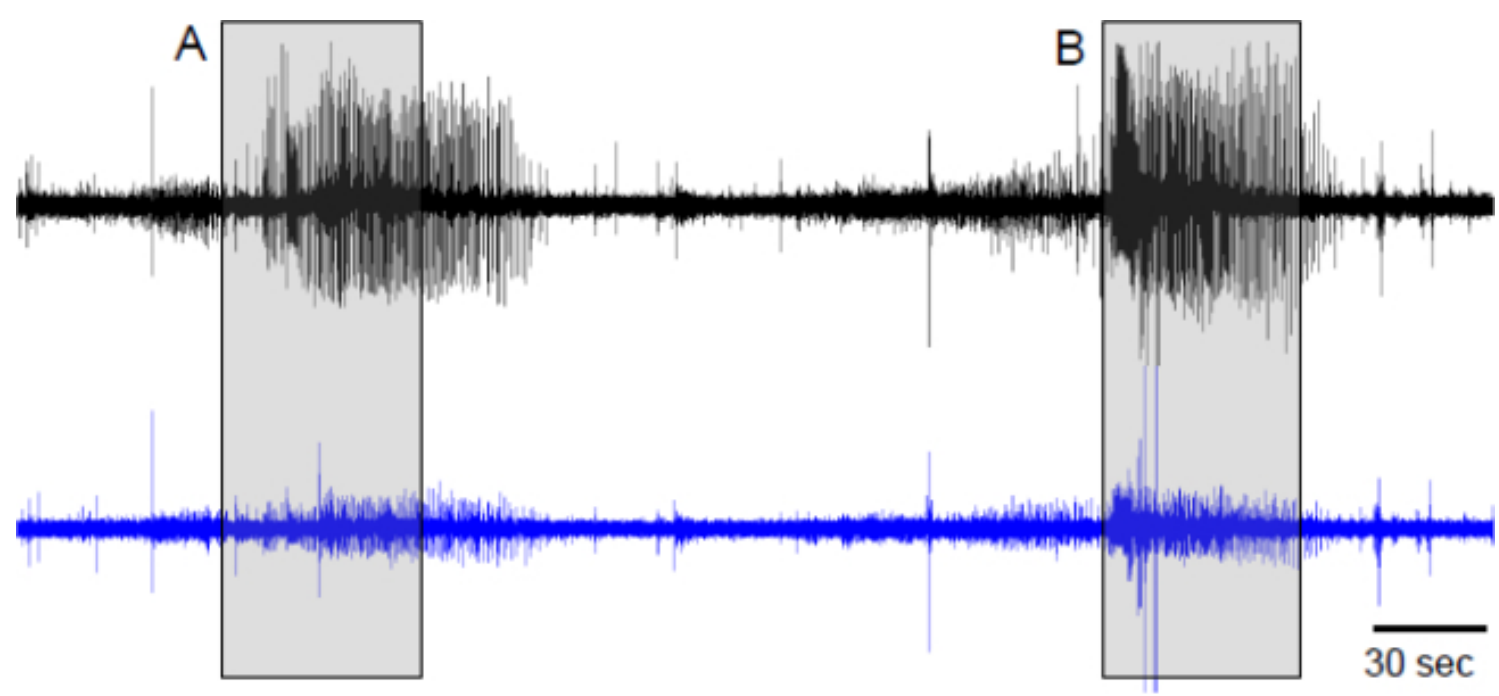

A
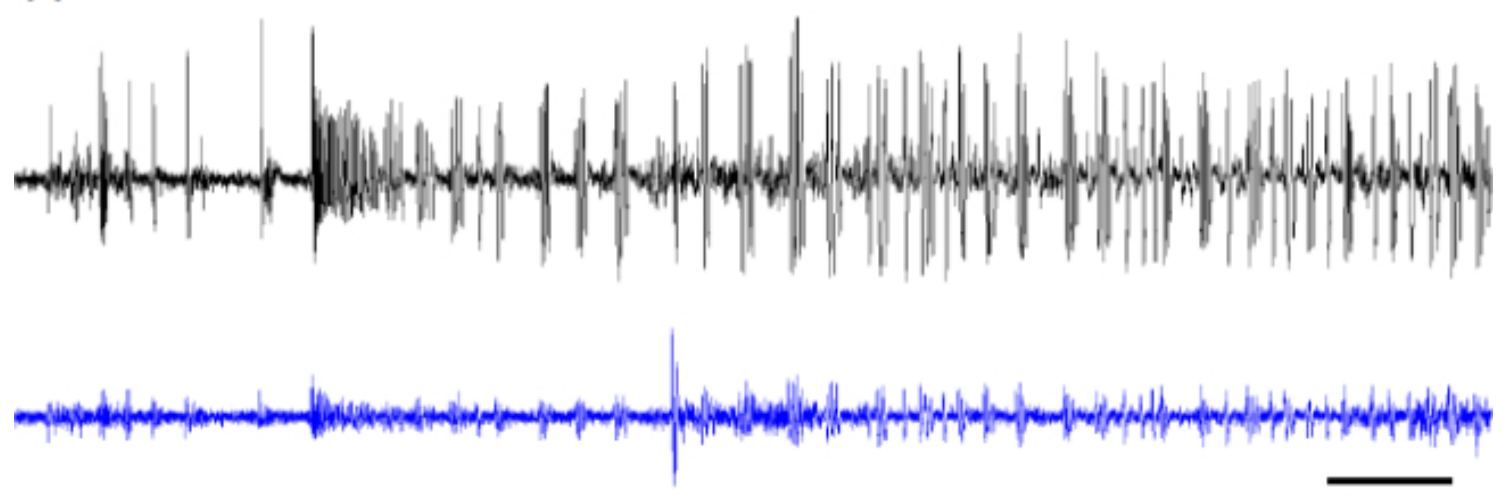

$3 \mathrm{sec}$

B
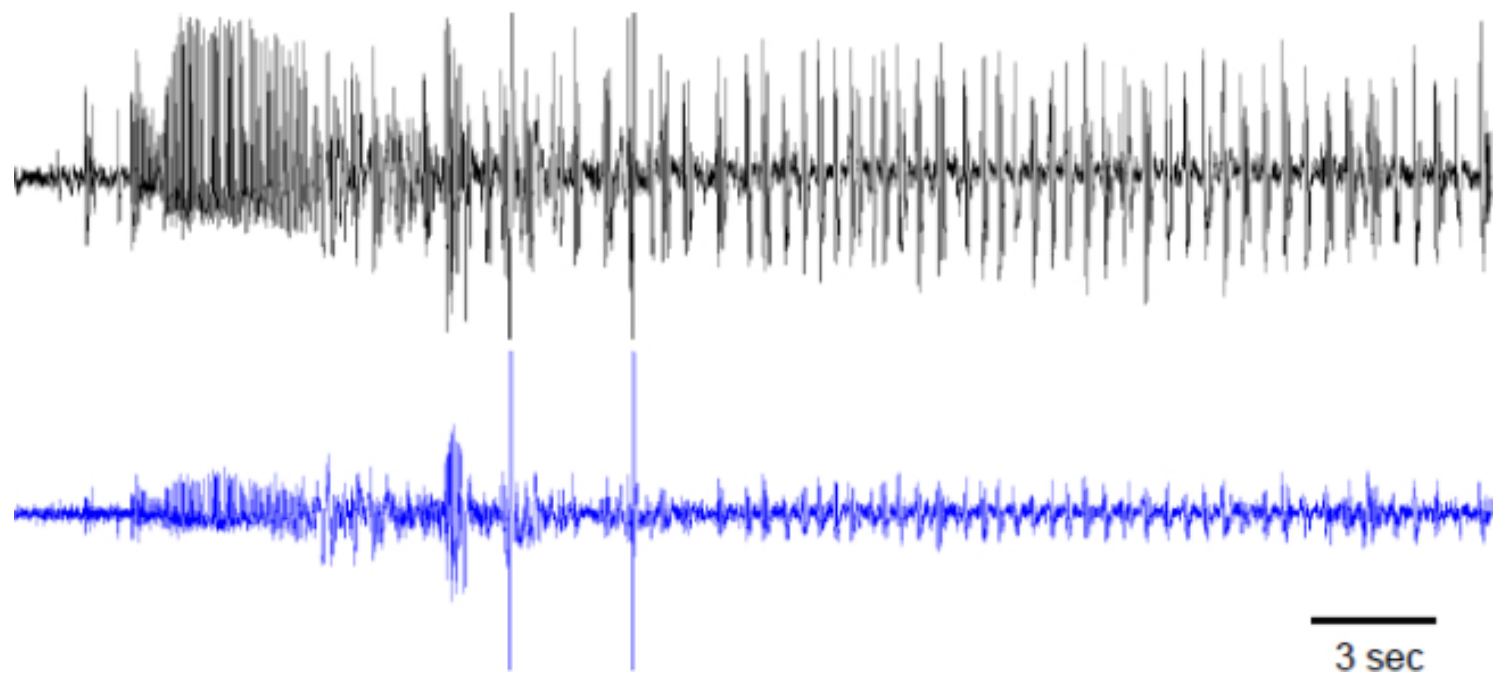

Figure 4: Dual-channel recording hypoxia-ischemia induced seizures with the telemetry system. Dual-channel recordings of abnormal EEG with the wireless telemetry in a P7 rat pup after carotid ligation (ischemia) during $8 \% \mathrm{O}_{2}$ treatment (hypoxia). (A) and (B), expanded views of the waveforms. Seizure activity is present in both hemispheres (black, blue) with substantial EEG suppression present in the hemisphere with ischemic infarct (blue). 
A B

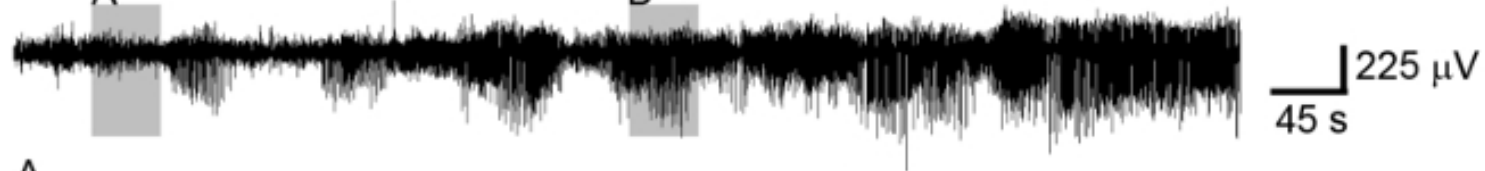

A

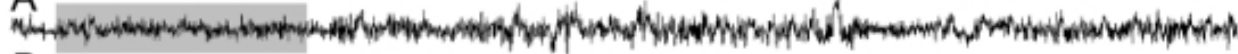

B

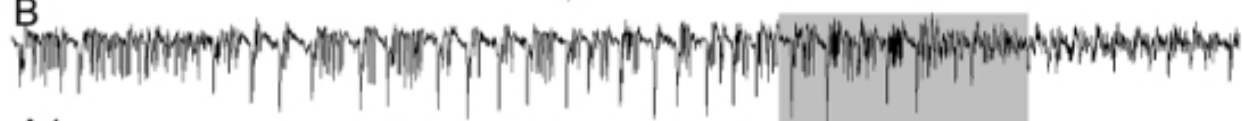

$2.5 \mathrm{~s}$

\section{A1}

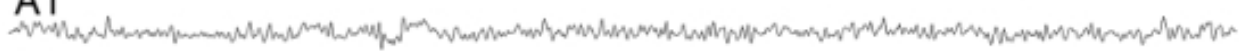

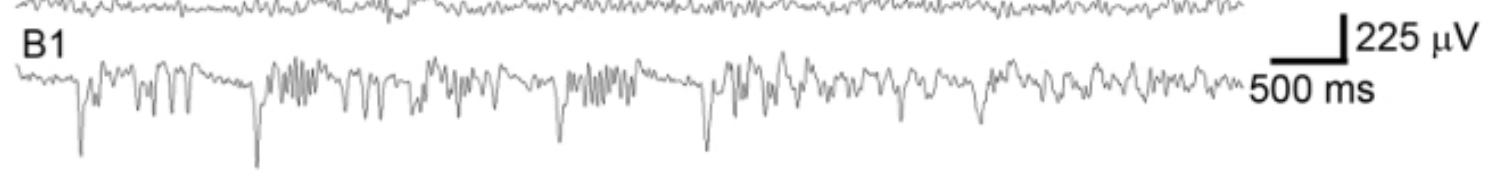

C

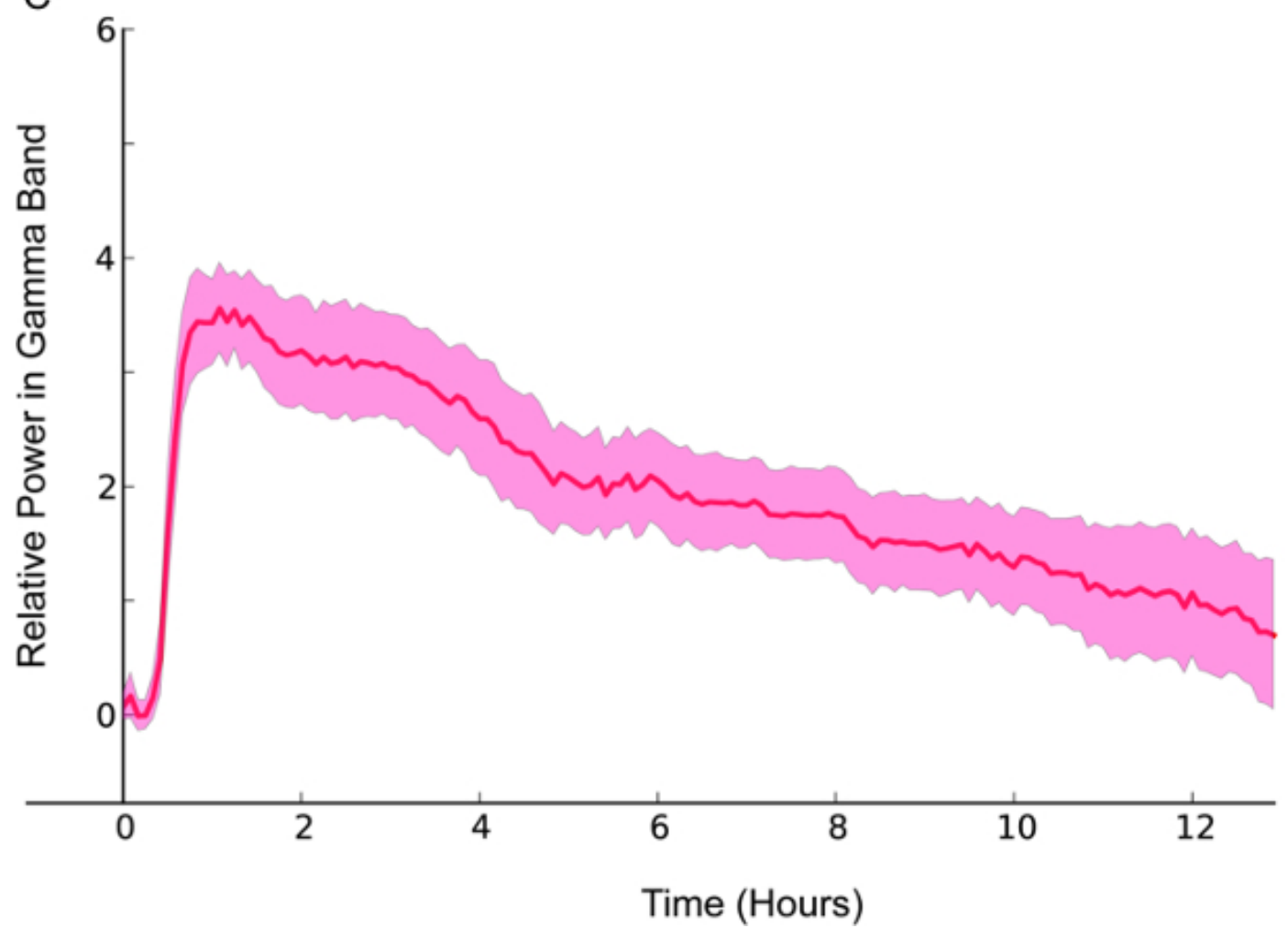

Figure 5: Recording status epilepticus in adult rats. Surface EEG recordings (i.e., dural) with the miniature wireless telemetry system in response to diisopropylfluorophosphate (DFP) treatment in an adult rat. The shaded periods in the top trace (A and $\mathbf{B})$ are expanded views of the waveforms in traces below. Data recorded with the wireless transmitter can then be analyzed in the frequency domain allowing statistical comparisons in a cohort of animals. (C) Data are the average and $95 \%$ confidence intervals of gamma band power $(20-60 \mathrm{~Hz})$ following DFPinduced status epilepticus $(\mathrm{N}=12)$ over $12 \mathrm{hr}$ after administration of DFP. 


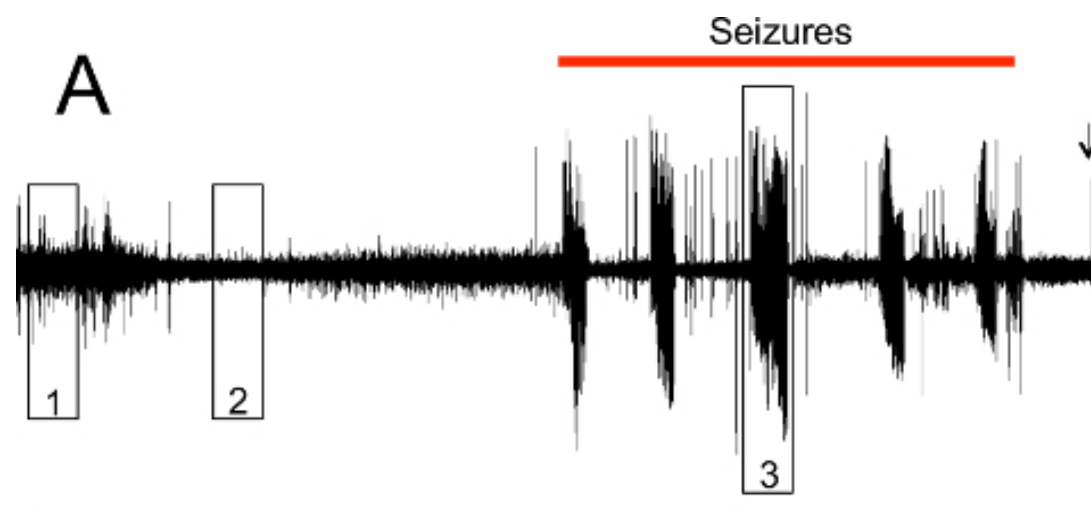

\section{Abnormal discharges}

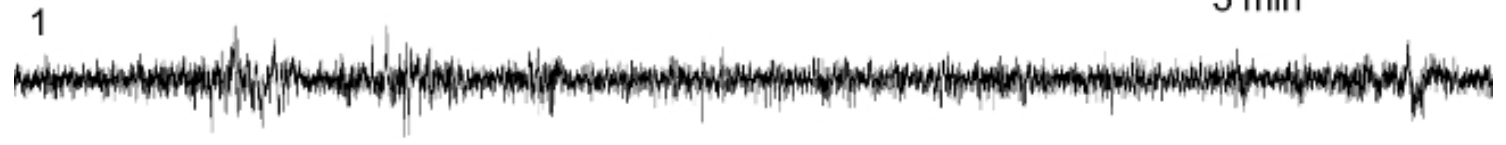

2

$10 \mathrm{sec}$
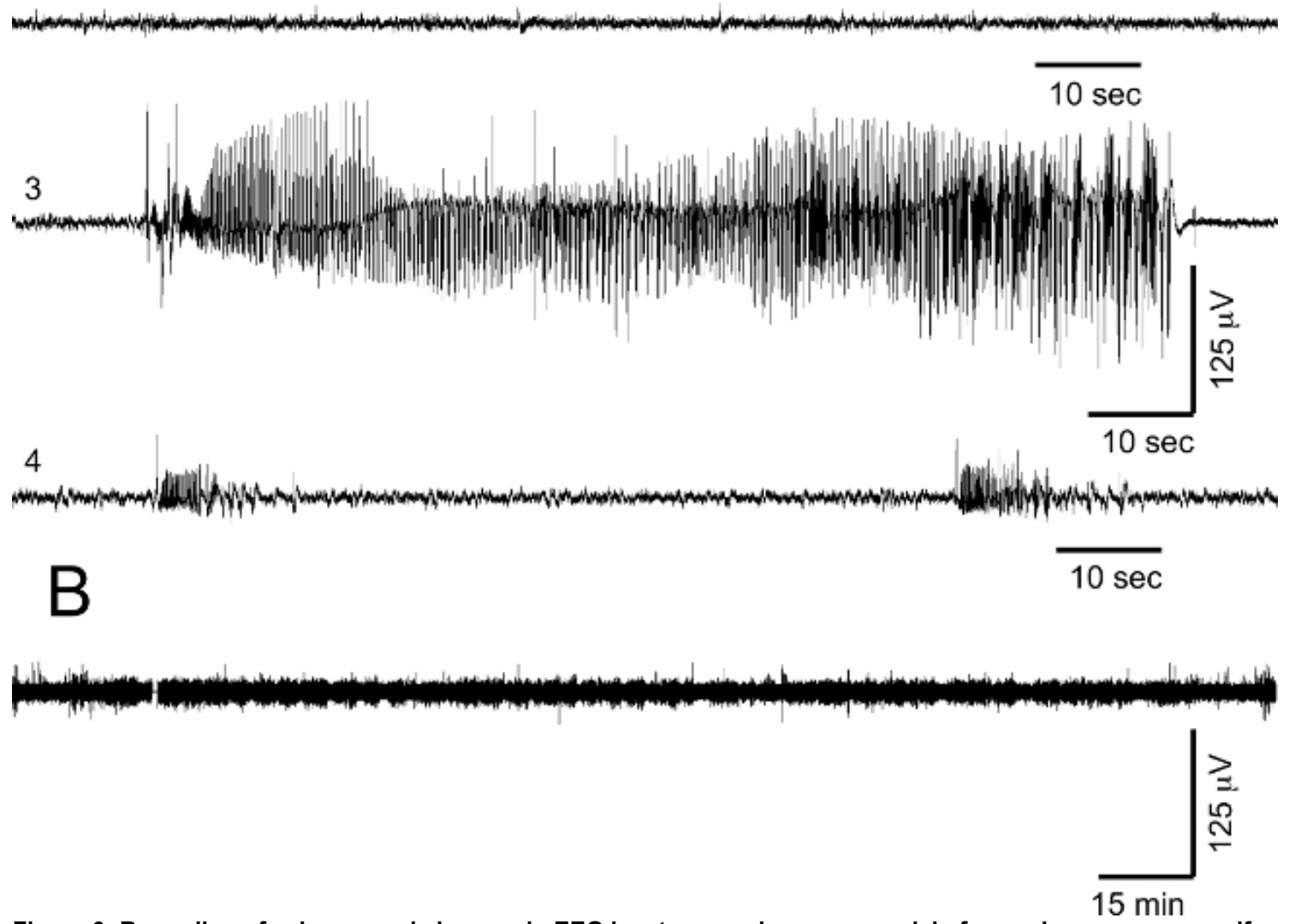

Figure 6: Recording of seizures and changes in EEG in a transgenic mouse model of vascular cavernous malformations. Here, we record from a transgenic mouse (A) that is undergoing seizure activity. At first, normal EEG pattern is present (1); immediately before the seizure there is a period of pre-ictal depression (2), which is followed by a cluster of five seizures (3). Following the seizures, abnormal ictal discharges are present in the signal (4). Control animal has no seizures and no abnormal EEG features (B).

\section{Discussion}

It can be very expensive to make long-term electrographic recordings in small-animal models of disease. By relying on simple electrical circuits and emphasizing low-power consumption, we have been able to create a transmitter system (Figures 1 and 2 ) that reduces the cost of long-term monitoring experiments. The total cost of a 6 month monitoring experiment could be as low as $\$ 470$, plus the cost of the animal $(\sim \$ 1.5$ animal per diem, $\$ 200$ transmitter). The small size of the transmitter permits continuous uninterrupted electrographic recordings in small-animal, preclinical models of human disease, which are very difficult to obtain with tethered or radio-frequency-based wireless recording systems (Figure 4). Finally, the skull-mounted nature of the transmitter reduces the surgery time and stress on the animal that can otherwise compromise an experiment. Here, we show proof-of-principle experiments from three different experimental models of seizures: perinatal hypoxia-ischemia ${ }^{13,}$ 
27,28 in a rat pup (Figure 4), DFP-induced status epilepticus (Figure 5) and seizures in a genetically-induced model of cavernous vascular malformations (Figure 6).

Possibly the most critical aspect for obtaining artifact-free, long-term electrographic recordings is to verify uninhibited electrode access to the cortical region of interest (Figure 4-6). This includes the common reference/ground electrode. Especially critical is attaching the transmitter to the skull for epidural EEG applications. During this process, it is possible to inadvertently coat the tip of the electrodes with cyanoacrylate given the very short length of the electrodes. Coating the electrodes in cyanoacrylate can attenuate the EEG signals or completely isolate them in the worst-case scenario. Similarly, lack of good electrical connection between the common reference/ground and the animal's brain will prevent correct operation of the differential amplifier in the transmitter, resulting in an electrically "noisy" signal output. Often, following surgery, goodquality signals can be compromised for up to $48 \mathrm{hrs}$ due to edema surrounding the burr holes in the skull. As the edema subsides, signals generally improve. This can be avoided by placing the electrodes on the surface of the skull without making burr holes. The consequences of this process are increased potential to coat the electrodes with cyanoacrylate, reduced high-frequency activity due to the low-pass electrical characteristics of the skull bone, and the potential to electrically isolate the common reference/ground rendering noise in the signals. Practicing correct placement of electrodes can be done with a thin piece of wood or veneer that mimics the thickness of the mouse or rat skull. The results presented in this manuscript illustrate the quality of recordings that can be obtained using wireless telemetry technology.

Surgical implantation using the method described herein can take as little as 10 min, depending on the complexity of the surgery. For surgical access to deep brain structures, such as the CA1 region of the hippocampus, it is best to attach the transmitter to a micromanipulator mounted to a stereotaxic frame. The micromanipulator will provide the surgeon with the accuracy to implant the transmitter according to published stereotaxic coordinates in the atlas of the mouse ${ }^{29}$ and rat ${ }^{30}$ brains. This can be done by simply tacking a piece of hypodermic needle tubing to the transmitter with cyanoacrylate and then mounting the hypodermic needle in the micromanipulator. Micromanipulator control of $x, y$, and $z$ coordinates will provide additional stability when mounting the transmitter to the skull prior to suturing the skin closed. The addition of bone screws around the perimeter of the transmitter can help anchor the transmitter to the skull, although they are not necessary. Bone screws could be effective, however, in certain animal models of seizures and epilepsy, such as the lithium-pilocarpine-treated adult rat. These animals tend to have spontaneous convulsive seizures with intense motor activity that may damage the transmitter during the seizure. Additional complexity could be added to these experiments. For example, the transmitter is compatible with many different models of traumatic brain injury, such as controlled cortical impact ${ }^{31}$. The durability of the transmitter device was tested by implanting animals with transmitters at P7, and then housing them in the animal facility. After 12 months, most of the implants remained intact on the skull. When animals were euthanized, the skulls appeared to be normal and the transmitter was embedded in the skull bone, requiring significant force to extract it. Take care when deep brain structures are studied; as the brain grows, and the electrodes remain stationary, the final position of the electrodes would be expected to change. For the techniques described here, the electrodes were typically positioned above the dura, which allowed both the brain and skull to grow and for electrodes to remain in their original positions. The limiting factor in how long the transmitter can be used is the battery size (i.e. until the battery runs out)

A self-contained monolithic design (i.e. the transmitter is embedded in hard epoxy) of the transmitter's housing lends itself to use with immature pups housed with the dam and their littermates. Often, co-housing implanted animals with wired tethers results in the destruction of the implanted hardware or cannibalization of the pups by the dam. The smooth-walled shape of the transmitter allows for implantation with virtually no hardware failure or loss of pups due to cannibalization.

The low-power capacitive-coupled transmission scheme in this system lends itself well for use in many different experimental scenarios such as placement in a temperature-controlled incubator or even for use with experimental apparatuses, such as plethysmography chambers. Likewise, the shape of the receiver antenna can be manipulated to fit in many different behavioral environments, such as the elevated T-maze.The range of the transmitter is only a few inches as the transmitter must be able to "drive" (capacitively couple to) the receiver antenna and relies on the animal to be housed in standard rodent caging or suitable cage covered by the receiver antenna surface area. The transmitter acts as one pole of an electric field whereas the body of the animal acts as another pole. The field is such that certain orientations, such as $90^{\circ}$ out-of-phase with the receiver antenna, will fail to drive the receiver antenna resulting in a "drop out". This is a rather rare occurrence. Future work with this technology will allow recording from multiple animals in the same cage that will reduce boarding costs and allow for social interaction. Further developments will involve increasing the number of channels including temperature, and electromyogram. The current design of the device provides a bandwidth that is optimized for recoding of classical EEG bands, which is not suitable for recording of fast ripples or high-frequency oscillations. In the future, the device can be modified to record the high-frequency components of the signal, but at the cost of battery life. Different types of transducers will include blood pressure and pressure-volume, and even construction of a transmitter electrode arrangement to user-specified dimensions according to stereotactic coordinates of the desired brain structures.

\section{Disclosures}

Drs. Lehmkuhle and Dudek have a financial interest in Epitel, Inc., designers of the Epoch wireless biopotential recording system.

Acknowledgements

This work was funded through the National Institute of Neurological Disorders and Stroke R43/R44 NS064661.

1. Boylan, G. B., Stevenson, N. J., Vanhatalo, S. Monitoring neonatal seizures. Semin. Fetal Neonatal Med. 18, (4), 208-208 (2013).

2. Panzica, F., Varotto, G., Rotondi, F., Spreafico, R., Franceschetti, S. Identification of the Epileptogenic Zone from Stereo-EEG Signals: A Connectivity-Graph Theory Approach. Front Neurol. 6, (4), 175 (2013).

3. Arciniegas, D. B. Clinical electrophysiologic assessments and mild traumatic brain injury: state-of-the-science and implications for clinical practice. Int J Psychophysiol. 82, (1), 41-52 (2011). 
4. Mizrahi, E. M., Kellaway, P. Cerebral concussion in children: assessment of injury by electroencephalography. Pediatrics. 73, (4), 419-425 (1984).

5. Pisarenco, I., Caporro, M., Prosperetti, C., Manconi, M. High-density electroencephalography as an innovative tool to explore sleep physiology and sleep related disorders. Int J Psychophysiol. S0167-8760, (14), 3-8 (2014).

6. Konadhode, R. R., et al. Stimulation of MCH neurons increases sleep. J. Neurosci. 33, (25), 10257-10263 (2013).

7. Bertram, E. H., Williamson, J. M., Cornett, J. F., Spradlin, S., Chen, Z. F. Design and construction of a long-term continuous video-EEG monitoring unit for simultaneous recording of multiple small animals. Brain Res. Protoc. 1-2, (1), $85-97$ (1997).

8. Stables, J. P., et al. Therapy discovery for pharmacoresistant epilepsy and for disease-modifying therapeutics: Summary of the NIH/NINDS/ AES Models II Workshop. Epilepsia. 44, (12), 1472-1478 (2003).

9. White, A. M., et al. Efficient unsupervised algorithms for the detection of seizures in continuous EEG recordings from rats after brain injury. J. Neurosci. Methods. 152, (1-2), 255-266 (2006).

10. Bertram, E. H., Cornett, J. F. The ontogeny of seizures in a rat model of limbic epilepsy: evidence for a kindling process in the development of chronic spontaneous seizures. Brain Res. 625, (2), 295-300 (1993).

11. Bertram, E. H., Cornett, J. F. The evolution of a rat model of chronic spontaneous limbic seizures. Brain Res. 661, (1-2), 157-162 (1994).

12. Williams, P. A., et al. Development of spontaneous recurrent seizures after kainate-induced status epilepticus. J. Neurosci. 29, (7), 2103-2112 (2009).

13. Kadam, S. D., White, A. M., Staley, K. J., Dudek, F. E. Continuous electroencephalographic monitoring with radio-telemetry in a rat model of perinatal hypoxia-ischemia reveals progressive post-stroke epilepsy. J. Neurosci. 30, (1), 404-415 (2010).

14. Galanopoulou, A. S. Basic mechanisms of catastrophic epilepsy -- overview from animal models. Brain Dev. 35, (8), 748-756 (2013).

15. Lerche, H., et al. Ion channels in genetic and acquired forms of epilepsy. J Physiol. 591, (Pt 4), 753-764 (2013).

16. Rossignol, E., et al. WONOEP appraisal: new genetic approaches to study epilepsy). Epilepsia. 55, (8), 1170-1186 (2014).

17. Westmark, C. J., et al. Reversal of fragile X phenotypes by manipulation of AßPP/A 3 levels in Fmr1KO mice. PLoS One. 6, (10), e26549 (2011).

18. Sukhotinsky, I., et al. Optogenetic delay of status epilepticus onset in an in vivo rodent epilepsy model. PLoS One. 8, (4), e62013 (2013).

19. Krook-Magnuson, E., Armstrong, C., Oijala, M., Soltesz, I. On-demand optogenetic control of spontaneous seizures in temporal lobe epilepsy. Nat Commun. 4, 1376 (2013).

20. Paz, J. T., et al. Closed-loop optogenetic control of thalamus as a tool for interrupting seizures after cortical injury. Nat Neurosci. 16, (1), 64-70 (2013).

21. Monod, N., Pajot, N., Guidasci, S. The neonatal EEG: statistical studies and prognostic value in full-term and pre-term babies. Electroecephalogr Clin Neurophysiol. 32, (5), 529-544 (1972).

22. Deshpande, L. S., Carter, D. S., Blair, R. E., DeLorenzo, R. J. Development of a Prolonged Calcium Plateau in Hippocampal Neurons in Rats surviving Status Epilepticus Induced by the Organophosphate Diisopropylfluorophosphate. Toxicol Sci. 116, (2), $623-631$ (2010).

23. Todorovic, M. S., Cowan, M. L., Balint, C. A., Sun, C., Kapur, J. Characterization of status epilepticus induced by two organophosphates in rats. Epilpsy Res. 101, (3), 268-276 (2012).

24. Lehmkuhle, M. J., et al. A simple quantitative method for analyzing electrographic status epilepticus in rats. J. Neurophysiol. 101, (3), 1660-1670 (2009).

25. Zayachkivsky, A., Lehmkuhle, M. J., Fisher, J. H., Ekstrand, J. J., Dudek, F. E. Recording EEG in immature rats with a novel miniature telemetry system. J. Neurophysiol. 109, (3), 900-911 (2013).

26. Pouliot, W., et al. A comparative electrographic analysis of the effect of sec-butyl-propylacetamide on pharmacoresistant status epilepticus. Neuroscience. 12, (231), 145-156 (2012).

27. Levine, S. Anoxic-ischemic encephalopathy in rats. Am J Pathol. 36, 1-17 (1960).

28. Vannucci, R. C., Vaccucci, S. J. A model of perinatal hypoxic-ischemic brain damage. Ann N Y Acad Sci. 835, 234-249 (1997).

29. Paxinos, G., Franklin, K. The Mouse Brain in Stereotaxic Coordinates. Academic Press Waltham, MA (2012).

30. Paxinos, G., Watson, C. The Rat Brain in Stereotaxic Coordinates. Academic Press Waltham, MA (2013).

31. Bolkvadze, T., Pitkanen, A. Development of post-traumatic epilepsy after controlled cortical impact and lateral fluid-percussion-induced brain injury in the mouse. J. Neurotrauma. 29, (5), 789-812 (2012). 